Full length article

\title{
Stiffness degradation of composite skin fields due to strength and buckling onset
}

\author{
Tanja Führer \\ German Aerospace Center Institute of Composite Structures and Adaptive Systems, Lilienthalplatz 7, 38108 Braunschweig, Germany
}

\section{A R T I C L E I N F O}

\section{Keywords:}

Composite structures

Failure criteria

Optimisation

\begin{abstract}
A B S T R A C T
Suitable and fast calculation methods with sufficient accuracy are required to optimise large composite structures. The present paper introduces a progressive stiffness degradation analysis (PSDA), which computes skin buckling onset and strength failure initiation of skin fields separated by stiffeners, as well as the subsequent damage propagation using closed form solutions. This constitutes a simplified, but much faster approach compared to state of the art progressive failure analyses, which incorporate finite element simulations. Therefore, the computational effort can be reduced. This paper illustrates the process of the PSDA and then verifies this simplified approach by means of one example.
\end{abstract}

\section{Introduction}

Complex multi-disciplinary optimisation chains, e.g. aircraft, gas turbine or helicopter design processes, have to be efficient in terms of computational costs (see Kroll et al. [27]). For the structural evaluation, different designs and various parameters have to be considered in order to exploit the potential of the structure. To this end, it is not necessary to model all physical details. Within the design process, suitable calculation methods are needed to analyse the structural behaviour. Thus, the evaluation of mechanical stresses via failure onset (strength failure and buckling failure) is performed. Typically, this process leads to a conservative and robust structural design, since no damages within the structures are allowed and redundant load paths are ignored. The accurate and efficient calculation of damage propagation up to ultimate laminate or structural failure has a potential for weight reduction. The analysis of the damage propagation needs to be modelled so as to obtain the stiffness reduction after ply failure and skin buckling onset. This in turn allows accounting for possible load redistribution. Consequently, the load carrying reserve can be exhausted and therefore the weight of the composite structure can be minimised. The application of this method can lead to adapted or reduced safety factors.

Prediction of failure onset and propagation can be done utilizing the finite element method (FEM) (e.g. see Oh et al. [32], Daghia and Ladevèze [19], Adden and Horst [1], Anyfantis and Tsouvalis [3]). Compared to the closed form solutions, the FEM more accurately computes the material behaviour after failure onset, e.g. geometric nonlinearity effects can be taken into account. Additionally, complex geometries can be considered and accurate solutions for a wide variety of problems can be achieved. The disadvantage of FEM approaches is the fact that it leads to high computational costs when large structures are optimised in a good quality solution.

The B0S0R5 computer program (see Bushnell $[8,9]$ ), based on the finite different energy method, is used to analyse segmented and branched shells with discrete ring stiffeners and multi-material constructions. B0S0R5 incorporates large deflection axisymmetric and small deflection non-symmetric analyses as well as the buckling analysis with axisymmetric and non-symmetric prestress. However, only laminates with up to 6 layers can be modelled. The minimum weight of a given structure can be computed using the program PANDA, which is used to optimise composite, stiffened, elastic-plastic panels (see Bushnell [10]). Thereby, the gradient based optimisation process includes stress and strain constraints as well as local buckling constraints. PANDA was superseded by PANDA2 (see Bushnell and Bushnell [11]), which also accounts for local post buckling behaviour and laminated composite panels. Additionally, it is possible to use closed form solutions when computing the buckling loads. The optimum design of composite structures can also be achieved using the tool VICONOPT (see Butler and Williams [12] as well as Watson et al. [45]), which is based on the exact strip theory assuming a continuous distribution of stiffness over the structure (infinitely long panels). The structure is optimised considering buckling and material strength constraints. Additionally, the post buckling stability of the stiffened plate can be computed. When coupled with MSC NASTRAN, VICONOPT provides a fast tool for multilevel optimisation of aerospace structures, such as composite aircraft wings (see Fischer et al. [20]). Both preliminary design tools VICONOPT and PANDA2 require less computational costs

E-mail address: Tanja.Fuehrer@dlr.de.

URL: http://www.dlr.de/fa. 
than BOSOR5, since the latter performs nonlinear static and stability analyses.

All three programs (BOSOR5, VICONOPT, PANDA2), which are mentioned above, do not account for material degradation after fibre or matrix failure, which can further reduce the weight of the structure. The material degradation after strength failure is considered within the proposed progressive stiffness degradation analysis (PSDA), which predicts skin buckling onset and the initiation of strength failure of skin fields separated by stiffeners (i.e. panels of an aircraft fuselage). Additionally, the material behaviour of composites after failure onset due to skin buckling and strength failure is taken into account. Failure initiation and damage propagation are also calculated with closed form solutions. To calculate the structural response, including the stresses within each finite shell element, the PSDA incorporates the solver within the software LAGRANGE, which is an in house tool of Airbus Defense \& Space. ${ }^{1}$ The software enables the analysis and optimisation of large structures, such as fuselage barrels [30]. It was applied successfully within several civil and military projects (see Schuhmacher et al. [41], Daoud and Calomfirescu [17] and Calomfirescu et al. [14]). The incorporated linear finite element solver is similar to the commercial software MSC NASTRAN. The sizing process is performed considering the major strength and buckling constraints. Within the gradient based optimisation process of LAGRANGE, the objective function, which is generally the structural weight, is minimised respecting the given constraints. The latter are for example reserve factors derived from strength and analytical buckling analyses or manufacturing constraints. Thereby, the derivatives for the gradient based optimisation can be computed numerically or analytically. The analytical approach, however, is mostly used for large structures. The initial design can be generated using the commercial software PATRAN or HYPERMESH.

In addition to the stresses within each finite shell element, LAGRANGE also computes the stresses where skin buckling onset occurs. Further details are given in the LAGRANGE handbook [30] and in Führer [21].

In the following section, an overview of the state of the art stress based failure criteria and degradation methods are given. Additionally, an overview of the buckling behaviour of composite structures is provided. The process of the PSDA, including the degradation of stiffener separated skin fields due to skin buckling onset and strength failure initiation, is discussed in the following Section 2. The degradation approach after skin buckling onset within the PSDA introduces a simplified approach compared to more sophisticated non-linear FEM analyses. To verify this method, an example incorporating two different loading conditions is calculated using the commercial software ABAQUS and the PSDA. The results from both analyses are discussed in Section 4. In the last section the paper is concluded.

\section{Failure modes of composite structures}

\subsection{Stress based failure criteria}

Compared to metal structures, composite materials have not uniform properties. To calculate failure onset and propagation, certain strength parameters are needed, which are used as a threshold to determine the load carrying capability. If the occurring load reaches the limit of the load carrying capability, material strength failure occurs. Within one laminae fibre or matrix failure (cracks, delaminations) can occur. The corresponding reserve factor can be calculated using numerous stress based failure initiation criteria, which are available in established literature. Nahas [31] summarises several limit failure theories without interaction (e.g. maximum stress criterion) and interaction criteria (e.g. Hill criterion). Examples for fracture plane-based criteria are the Puck criterion, reviewed in Puck [35] and Puck and the

\footnotetext{
${ }^{1}$ Former CASSIDIAN Air Systems.
}

Schürmann [36] and the Cuntze criterion [16]. The Puck criterion considers the fracture plane angle, which can be calculated analytically for the two dimensional case (see [25]). The Cuntze criterion contains five failure modes and an interaction between all modes, which is expressed through invariants.

The Puck and the Cuntze methods have been implemented within the PSDA, because they are more sophisticated than other sudden reduction methods; such as the method by Sun and Tao. Compared to more complex methods by Reddy and Reddy [38], the implementation effort and the computational cost are much lower. It must be noted that the strength evaluation due to compressive loading is not taken into account because the paper only considers thin structures where the effect is negligible.

Other failure initiation criteria are energy based criteria (see Barakat and Abu-Farsakh [4] and Wolfe and Butalia [48]), tensor polynomial failure criteria (see Craddock and Champagne [15]) and fracture based failure criteria, such as the virtual crack closure technique (see Krueger [28]). However, due to their high implementation effort the three criteria mentioned above are not considered within the PSDA. Several stress based failure criteria are also summarised in [21].

\subsection{Stability phenomena}

In addition to strength failure, the load carrying capability of thinwalled structures can decrease due to stability phenomena when subjected to compression or shear loading. In the aircraft design process, two types of structures are typically evaluated with respect to stability: skin fields on a wing that are located between two ribs and two spars, and skin fields in the fuselage that are between two stringers and two frames. To determine whether the stiffness within one skin field has to be degraded due to skin buckling onset, the load displacement curve can be inspected. Up to a certain point, the structure's behaviour, including its load displacement curve, is assumed to be linear. Beyond this point, a load increase leads to buckling deformations. This point, where the solution branches off from the primary solution is called bifurcation point. The load at the lowest bifurcation point is termed buckling load, which can be calculated by FEM, using an eigenvalue analysis or the more costly transient analysis. Therefore, the FEM based eigenvalue analysis requires a sufficiently fine mesh to consider local effects, which in turn leads to high computational costs. To overcome this issue, simplified analytical models are used. One of the first analytical approaches was developed by Bryan [6], who calculated the buckling load of a simply supported plate. Karman and Tsien [44] performed several buckling analyses by means of the non-linear large deflection theory. Koiter [26] addressed the area surrounding the bifurcation point and developed a series expansion near the lowest critical eigenvalue of the respective structure.

In the present paper, the stresses that lead to skin buckling in one skin field are calculated by the software LAGRANGE. Here closed form solutions are already implemented, which are based on the Weaver (see $[46,47])$ and ESDU $[18]$ methods. The necessary loads and material data are defined by the user within the PSDA. The resulting buckling stresses are passed to the PSDA in addition to the corresponding stresses within each finite shell element. If the reserve factor due to buckling (computed by dividing the actual stress by the buckling stress) reaches the threshold, skin buckling ensues within the corresponding skin field.

\subsection{Postbuckling}

In order to further exploit the load carrying reserve after skin buckling onset, the post buckling behaviour within the sizing and optimisation process of the structure has to be considered. Within the present PSDA, certain parts of the ABD matrix of the failed skin field are reduced by a factor, which is determined from several nonlinear analyses. The procedure will be explained in detail in Section 3.1.

Boni et al. [5] implemented standard finite element codes to 
compute the post buckling behaviour of composite flat panels. Other methods use semi-empirical approaches to calculate the post buckling behaviour. Pevzner [33] incorporated the effective width method and Kuhn [29] the diagonal tension approach to overcome the disadvantage of high computational costs. Byklum et al. [13] proposed a semi-analytical model computing global buckling and post buckling of stiffened isotropic plates using large deflection plate theory and a global-local approach. Another approach was developed by Buermann [7], who uses the analytical continuum mechanics and numerical methods. Nevertheless, compared to the effective width method and the diagonal tension approach, the progressive stiffness degradation analysis considers various combinations of loading conditions. Due to the closed form solutions, the PSDA is even more computational efficient than the semi empirical approaches mentioned above.

In order to recalculate the structural model after the element stiffness matrix is reduced, the material properties are adapted in the LAGRANGE input file. Since the PSDA considers only a part of the complete load displacement behaviour in the post buckling regime, it is a simplified approach compared to more sophisticated nonlinear buckling analyses. However, regarding the computational costs, the PSDA is much faster compared to FEM analyses.

After skin buckling onset is checked within each skin field, the PSDA computes the reserve factors due to strength failure onset. As already mentioned, several stress based failure criteria are available. For each implemented stress based failure criterion several reserve factors have to computed, which in turn require the actual stresses in the observed finite element. The latter are defined by the user and depend on the respective material. The resulting stresses are passed to the PSDA, which calculates the respective reserve factors.

\subsection{Degradation of the model}

Because composite laminates may not fail completely after initial matrix or fibre failure, the material properties have to be changed after strength failure onset to consider the preliminary damage within the structure. The material property reduction can be done gradually or instantaneously. Garnich and Akula [22] and Rohwer [40] summarise several degradation methods. A degradation method, which implies a sudden reduction of material properties after matrix failure onset is proposed by Sun and Tao [43]. For fibre fracture, the longitudinal modulus is set to nearly zero. More sophisticated methods were proposed by Reddy and Reddy [39] and Phillips et al. [34]. Reddy and Reddy apply a constant stiffness reduction coefficient, which is established experimentally for a given laminate. The method developed by Phillips uses two and three dimensional finite elements and an additional interface is modelled between two layers.

Within the PSDA, the Puck (see [35]) method and two instantaneous degradation methods (see [21]) are implemented. All three methods, which were chosen due to their low implementation and computational effort, will be explained further on.

Depending on the degradation method, several elastic moduli are reduced. The resulting material properties are passed to the model input file and the model is recalculated. If no further strength failure or skin buckling onset is detected, the load is increased. This is done until the structure fails completely. Before the recalculation of the structural model, the increments of the mechanical stresses $\Delta \sigma$ and displacements $\Delta \mathrm{u}$ within the current load step are subtracted from their total values $\sigma$ and $\mathrm{u}$, respectively. This is done, because the stresses and strains after failure initiation have to be computed using the reduced material properties.

The results of the PSDA are, amongst others, the load displacement curve. Even though the chosen failure initiation and degradation criteria represent only a small selection from the existing methods, they do reflect the several levels of complexities.

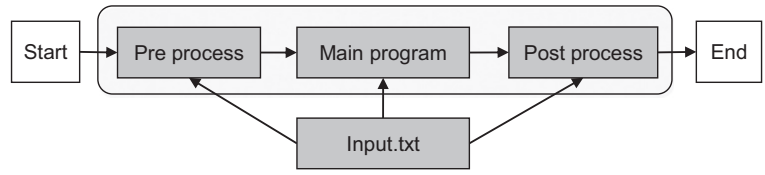

Fig. 1. Process of progressive stiffness degradation analysis (see [21]).

\section{Implementation of the PSDA}

The proposed PSDA is implemented using the object oriented programming language Python. It consists of three main parts (cf. Fig. 1). Firstly, the pre process reads the input file, which contains various parameters describing the structure (i.e. finite element dimensions, material properties, stacking sequence and ply thickness). Since several structural parameters can be adapted, it is possible to calculate various use cases and configurations. Additionally, a failure initiation and a degradation criterion are chosen.

Secondly, the main program calculates the structural response of the given structure. Thirdly, the post process contains the routines to plot the load displacement curves of the local structure. Here the sum of the external loads and the displacements from a predefined node in each step are used. The following sections describe the failure initiation and degradation routines within the main program.

\subsection{Integration of the buckling behaviour}

To consider the local post buckling behaviour in a linear analysis, the element stiffness properties of one skin field have to be reduced, if skin buckling onset is detected. Each skin field is meshed with one linear finite shell element. For the sample analysis, the software LAGRANGE has been employed. This software is used to optimise large structure in a preliminary design phase, which leads to a coarser mesh compared to the mesh of the converged solution. Fig. 2 depicts a skin field defined as being located between two stringers and two frames on the fuselage. The stiffeners on the edges of the skin field are modelled with finite beam elements.

The loads at which skin buckling ensues (critical skin buckling load $P_{\text {Buck}}$ ) are computed by LAGRANGE using the Paul Weaver and ESDU methods. Within the ESDU method the buckling modes are expressed by Taylor series [18]. The Weaver method uses closed form solutions to calculate buckling of long anisotropic flat plates. The uniaxial compression load case is computed using the following Eqs. (1)-(5) (see Weaver [47]), where $K_{X}$ represents the influence of the flexural/twist anisotropy on buckling plates and $\beta$ Eq. (3) the plate bending orthotropy. The plate bending anisotropy is described by $\gamma$ and $\delta$ Eqs. (4) and (5).

$\mathrm{N}_{\mathrm{X}}=\frac{\pi^{2}}{\mathrm{~b}^{2}} \mathrm{~K}_{\mathrm{X}} \cdot \sqrt{\mathrm{D}_{11} \mathrm{D}_{22}}$

$\mathrm{K}_{X}=2(1+\beta)+2\left(\beta+3+2 \gamma^{2}\right) \frac{(\gamma+3 \delta)^{2}}{(3+\beta)^{2}}-4\left(\delta+2 \gamma^{3}-\beta \gamma\right) \frac{(\gamma+3 \delta)^{3}}{(3+\beta)^{3}}$

$\beta=\frac{D_{12}+2 D_{33}}{\sqrt{D_{11} D_{22}}}$

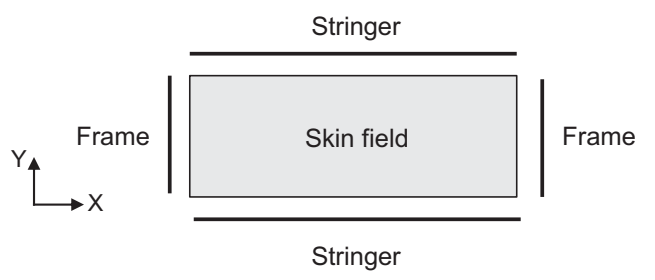

Fig. 2. Definition of skin field (see [21]). 
$\gamma=\frac{\mathrm{D}_{13}}{\sqrt[4]{\mathrm{D}_{11}^{3} \mathrm{D}_{22}}}$

$\delta=\frac{D_{23}}{\sqrt[4]{D_{22}^{3} D_{11}}}$

The shear load case is computed using the following Eqs. (6) and (7) (see Weaver [46]).

$\mathrm{N}_{\mathrm{XY}}=\frac{\pi^{2}}{\mathrm{~b}^{2}} \mathrm{~K}_{\mathrm{XY}} \cdot \sqrt[4]{\mathrm{D}_{11} \mathrm{D}_{22}^{3}}$

$\mathrm{K}_{\mathrm{XY}}=3.42+2.05 \beta-00.13 \beta^{2}-1.79 \gamma-6.89 \delta+0.36 \beta(2 \gamma+\delta)$

$$
-0.25(2 \gamma+\delta)^{2}
$$

Those solutions were verified by means of detailed finite element analyses and numerical solutions (see $[46,47]$ ). Compared to the ESDU method, the Weaver method computes each load where buckling ensues separately. Therefore, the ESDU method is used for combined loading. Both methods assume flat, simply supported skin fields, which leads to limitations within the PSDA. Skin fields with all edges simply supported underestimate the buckling loads, because an indefinitely high stiffness is employed. The actual edge boundary conditions of realistic skin fields are between simply supported and clamped. However, since studies showed that clamped edges lead to higher buckling loads compared to simply supported edges, the latter represents a more conservative approach.

After the stresses and buckling loads are calculated for each skin field, the results are passed to the PSDA. If the stress within the skin field exceeds the value of $\sigma_{\text {buckcrit }}$ (critical skin buckling stress), the corresponding stiffness properties are reduced by a degradation factor $\mathrm{f}_{\text {degbuck }}$, which is derived by a nonlinear analysis using the commercial software ABAQUS. For each skin field with different element properties and loading conditions, one ABAQUS analysis is performed automatically and prior to the PSDA. However, after the ABAQUS analysis is run for one specific skin field, the results including the degradation factor $f_{\text {degbuck }}$ can be used for each skin field with the same characteristics.

The element properties, which are varied, include the element dimensions, the material data, the ply thickness and the stacking sequence. Regarding the loading conditions, seven different load cases are considered, including uniaxial and biaxial compression load as well as shear load. Also a combination of shear and uniaxial compression load, shear and biaxial compression load, shear and uniaxial tension load and shear and biaxial tension load are taken into account.

All investigated skin fields are computed separately and are meshed much finer as it is with the PSDA. Within each nonlinear finite element analysis the element properties and the loading condition of the skin field are varied. Since all skin fields within LAGRANGE are non-stiffened, the same is assumed for the skin fields within the ABAQUS analyses. To match the closed form solution (Weaver and ESDU), with which skin buckling onset is calculated within LAGRANGE, the skin fields are simply supported and flat.

The result of each ABAQUS analysis is one load displacement curve, which represents the corresponding LAGRANGE skin field properties and loading condition of the global model. It is used to calculate the degradation factor $\mathrm{f}_{\text {degbuck }}$.

In Fig. 3, the relationship between the load displacement curve and $f_{\text {degbuck }}$ is illustrated. Before skin buckling ensues $\left(P_{\text {Buck }}\right)$, the stiffness properties remain unchanged $\left(f_{\text {degbuck }}=1\right)$. After the critical load $\left(\mathrm{P}_{\text {fail }}\right)$, where the skin field fails completely, is reached, the material properties are set to nearly $0\left(f_{\text {degbuck }} \approx 0\right)$. Between $P_{\text {Buck }}$ and $P_{\text {fail }}$ the degradation factor $f_{\text {degbuck }}$, representing the residual stiffness of the skin field after skin buckling onset, depends on the characteristics of the corresponding skin field.

The value of $f_{\text {degbuck }}$ is calculated dividing the value of the slope in the post buckling regime $\left(\mathrm{K}_{\text {post }}\right)$ by the slope in the pre buckling regime

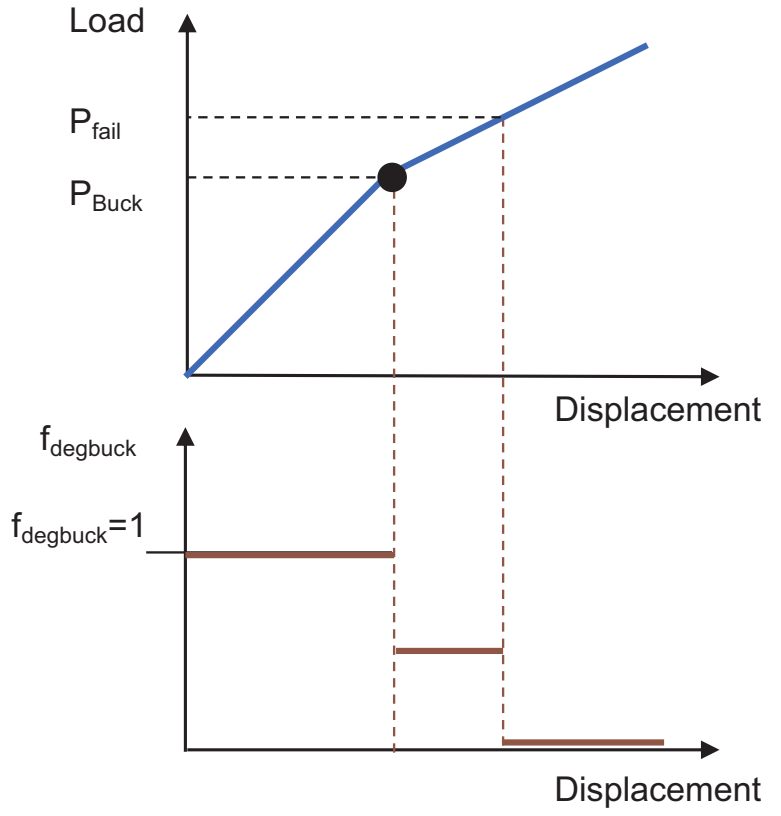

Fig. 3. Derivation of degradation factor $\mathrm{f}_{\text {degbuck }}$ from load displacement curve (see [21]).

(Kre) (Eq. (8) see [21]).

$\mathrm{f}_{\text {degbuck }}=\frac{\mathrm{K}_{\text {Post }}}{\mathrm{K}_{\text {Pre }}}$

The corresponding slopes $\mathrm{K}_{\text {Post }}$ and $\mathrm{K}_{\text {Pre }}$ are calculated using the Eq. (9) (see [21]), where the parameter $\mathrm{P}$ represents the load and $\mathrm{u}$ the displacement at the corresponding increment inc. For example, if the skin field is loaded with uniaxial compression in $\mathrm{x}$-direction, the load $\mathrm{P}_{\mathrm{X}}$ and the displacement $\mathrm{u}_{\mathrm{X}}$ are used to compute the slopes $\mathrm{K}_{\mathrm{Post}}$ and $\mathrm{K}_{\mathrm{Pre}}$ for the corresponding direction. For biaxial compression in $\mathrm{x}$-direction and $\mathrm{y}$-direction $\mathrm{K}_{\mathrm{PreX}}$ and $\mathrm{K}_{\mathrm{PreY}}$ are calculated separately, using $\mathrm{u}_{\mathrm{X}}$ and $\mathrm{P}_{\mathrm{X}}$ as well as $\mathrm{u}_{\mathrm{Y}}$ and $\mathrm{P}_{\mathrm{Y}}$, respectively.

$\mathrm{K}_{\mathrm{inc}}=\frac{\Delta \mathrm{P}_{\mathrm{inc}}}{\Delta \mathrm{u}_{\mathrm{inc}}}=\frac{\mathrm{P}_{(\text {inc }+1)}-\mathrm{P}_{(\mathrm{inc}-1)}}{\mathrm{u}_{(\text {inc }+1)}-\mathrm{u}_{(\text {inc-1) }}}$

To determine the displacements $\mathrm{u}_{\text {Pre }}, \mathrm{u}_{\mathrm{Buck}}$ and $\mathrm{u}_{\text {Post }}$, the increments at the corresponding points within the load displacement curve are

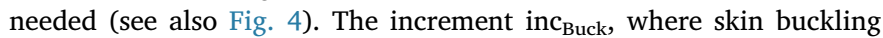
ensues, is obtained from the nonlinear load displacement curve. Here, the increment is taken, where the slope drops by $5 \%$. The increment inc $_{\text {Pre }}$ (corresponding to $\mathrm{P}_{\text {Pre }}$ and $\mathrm{u}_{\text {Pre }}$ ) is calculated by dividing inc Buck $_{\text {Buck }}$ by a factor of 2 . However, the increment in $c_{\text {Buck }}$ can be divided by any factor, since the slope in the pre buckling region remains constant. The

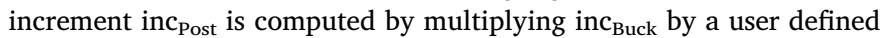
factor $\mathrm{f}_{\text {Post }}$ (Eq. (10)).

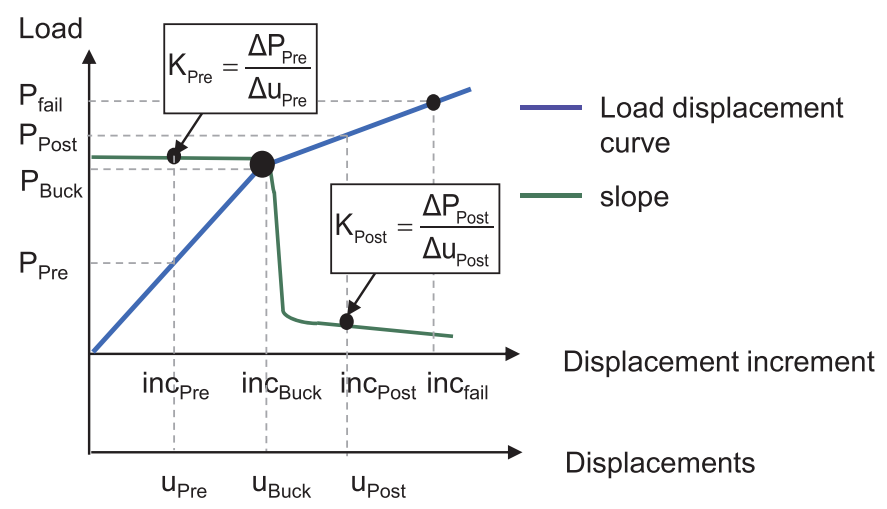

Fig. 4. Computation of different increments (see [21]). 


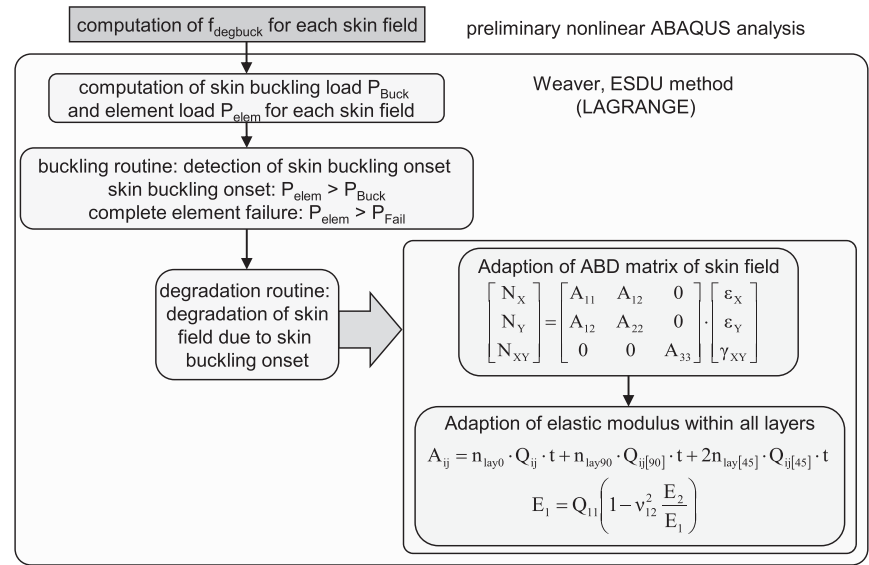

Fig. 5. Degradation due to skin buckling onset within the PSDA.

inc $_{\text {Post }}=$ inc $_{\text {Buck }} \cdot \mathrm{f}_{\text {Post }}$

The factor $f_{\text {Post }}$ has to be chosen between 1.1 and 1.6. This definition ensures a conservative design, because only a part of the post buckling regime in the vicinity of the buckling load $\mathrm{P}_{\mathrm{Buck}}$ is considered. For a combination of loading conditions, two degradation factors $f_{\text {degbuck } 1}$ and $\mathrm{f}_{\text {degbuck2 } 2}$ have to be calculated (see [21]). To compute the increment inc $_{\text {fail }}$, where the skin field fails completely, inc Buck $_{\text {Buck }}$ is multiplied by another user defined factor $f_{\text {fail }}$, which has to be greater than $f_{\text {Post }}$.

All relevant data computed by ABAQUS are stored in one text file, which is passed to the PSDA. The data within the text file includes the buckling factors $\mathrm{f}_{\text {degbuck }}$, the material data, the loading condition, the stacking sequence and dimensions of each individual skin field.

\subsection{Degradation process due to buckling}

The following Fig. 5 illustrates the degradation process due to skin buckling onset within the PSDA. If the element load $\mathrm{P}_{\text {Elem }}$ in one skin field exceeds $\mathrm{P}_{\text {buck }}$, then skin buckling onset is detected and the element properties are adapted. For combined loading conditions, such as shear and compression, the loads $\mathrm{P}_{\text {Elemx }}$ and $\mathrm{P}_{\text {ElemXY }}$ are compared to the buckling loads $\left(\mathrm{P}_{\text {buckX }}\right.$ and $\left.\mathrm{P}_{\text {buckXY }}\right)$ in the corresponding directions. The loading condition within each skin field is derived from the ratio of the element fluxes $\mathrm{N}_{\mathrm{X}}, \mathrm{N}_{\mathrm{Y}}$ and $\mathrm{N}_{\mathrm{XY}}$ (please refer to [21]). If the load within one skin field $\mathrm{P}_{\text {Elem }}$ exceeds the critical load $\mathrm{P}_{\mathrm{Fail}}$, then the skin field is considered to have failed completely.

In order to adapt the element properties of one skin field, the ABD matrix (based on the classical laminate theory) is reduced. The values within the $\mathrm{B}$ part of the ABD matrix are zero, since the present paper only considers symmetric layups. The observed loading conditions do not include moments $\left(M_{x}=M_{y}=M_{x y}=0\right)$ and only certain entries of the A matrix are multiplied by the computed degradation factor $\mathrm{f}_{\text {degbuck, }}$, or by two factors $f_{\text {degbuck1 } 1}$ and $f_{\text {degbuck2 }}$. The next section explains the degradation process in more detail using uniaxial compression as an exemplary loading condition. If skin buckling onset is detected, then the ABD matrix is reduced. Here the load in y-direction and the resulting strain in y-direction are zero. This also applies to the shear load and the resultant strain. Consequently, the entry $A_{11}$ is changed $\left(A_{11}\right.$ is multiplied by $\mathrm{f}_{\text {degbuck }}$, see Eq. (11).

$\left[\begin{array}{c}\mathrm{N}_{\mathrm{X}} \\ 0 \\ 0\end{array}\right]=\left[\begin{array}{ccc}\mathrm{A}_{11} \cdot \mathrm{f}_{\text {degbuck }} & 0 & 0 \\ 0 & 0 & 0 \\ 0 & 0 & 0\end{array}\right] \cdot\left[\begin{array}{c}\varepsilon_{\mathrm{X}} \\ 0 \\ 0\end{array}\right]$

For biaxial compression, the entries $\mathrm{A}_{11}$ and $\mathrm{A}_{22}$ are adapted, since the shear load and resultant shear strain are zero. The entry $A_{33}$ is changed when shear loading is most critical, because the loads and resultant strains in $\mathrm{x}$ - and $\mathrm{y}$-direction (the definition of the $\mathrm{x}$ - and $\mathrm{y}$ directions can be taken from Fig. 2) are zero. For combined shear and compression as well as the combination of shear and tension, the entries $A_{11}$ and $A_{33}$ are adapted. Here the strain and load in the y-direction remains zero. The A matrix for each skin field can be changed by adapting the LAGRANGE input file. The latter contains the material properties (i.e. elastic moduli) for each skin field. Therefore, the degradation factors are applied to the elastic modulus $E_{1}$. Depending on which part of the A matrix has to be adapted, the factor for the elastic modulus is calculated utilizing $f_{\text {degbuck }}$, or $f_{\text {degbuck1 }}$ and $f_{\text {degbuck2 }}$. Regarding uniaxial compression for example, the elastic modules in fibre direction $E_{1}$ of all layers are adapted using the following Eq. (12) (for a stacking sequence including $0^{\circ}, 90^{\circ}$ and $+/-45^{\circ}$ layers). Here $\mathrm{n}_{\text {lay }}$ presents the number of layers in the respective direction, $Q$ the ply stiffness matrix and the thickness. The following Eqs. 12-14 are taken from Altenbach [2].

$\mathrm{A}_{11}=\mathrm{n}_{\text {lay } 0} \cdot \mathrm{Q}_{11} \cdot \mathrm{t}+\mathrm{n}_{\text {lay } 90} \cdot \mathrm{Q}_{11[90]} \cdot \mathrm{t}+2 \mathrm{n}_{\text {lay }[45]} \cdot \mathrm{Q}_{11[45]} \cdot \mathrm{t}$

$\mathrm{Q}_{11}=\frac{\mathrm{A}_{11}-\left(\mathrm{n}_{\text {lay } 90} \cdot \mathrm{Q}_{11[90]^{\prime}} \cdot \mathrm{t}+2 \mathrm{n}_{\text {lay }[45]^{\prime}} \mathrm{Q}_{11[45]^{\prime}} \mathrm{t}\right)}{\mathrm{n}_{\text {lay } 0} \cdot \mathrm{t}}=\frac{\mathrm{A}_{11}-A_{\text {const. }}}{\mathrm{n}_{\text {lay } 0} \cdot \mathrm{t}}$

$\mathrm{Q}_{11}=\frac{\mathrm{E}_{1}}{1-v_{12}^{2} \frac{\mathrm{E}_{2}}{\mathrm{E}_{1}}}$

Combining Eqs. (13) and (14), the elasticity modulus in the $0^{\circ}$ direction can be computed by Eq. (15).

$\mathrm{E}_{1}=\frac{\mathrm{A}_{11}-A_{\text {const. }}}{2 \mathrm{n}_{\text {lay } 0} \cdot \mathrm{t}} \pm \sqrt{\left(\frac{\mathrm{A}_{11}-A_{\text {const. }}}{2 \mathrm{n}_{\text {lay } 0} \cdot \mathrm{t}}\right)^{2} \frac{1}{4}-\left(\frac{\mathrm{A}_{11}-A_{\text {const. }}}{\mathrm{n}_{\text {lay } 0} \cdot \mathrm{t}} \cdot v_{12}^{2} \mathrm{E}_{2}\right)}$

The derivations for all elastic modulis are described in [21]. It is also possible to extend the PSDA to unsymmetrical stacking sequences.

\subsection{Progressive stiffness degradation analysis considering strength and stability}

The overall process of the PSDA is depicted in Fig. 6. After each structural analysis (using the finite element solver within LAGRANGE), all resulting loads of all skin fields, as well as all buckling loads are passed to the buckling routine within the PSDA. If the load within the element $\mathrm{P}_{\text {Elem }}$ exceeds the buckling load $\left(\mathrm{P}_{\text {Buck }}\right)$, skin buckling onset is detected and the element properties of the corresponding skin field are degraded. Afterwards, the structural response is recalculated by LAGRANGE. Prior to the recalculation, the stresses $\Delta \sigma$ and the displacements $\Delta \mathrm{u}$ within the current iteration are subtracted from their total values $\sigma$ and $u$ ("Subtraction of stresses" in Fig. 6). This is necessary since the structure has to be recalculated using reduced material properties due to the degraded skin fields.

If no further stiffness degradation due to skin buckling failure occurs in the next iteration step, the strength routine is performed. Fig. 7 illustrates the initiation of strength failure and the corresponding degradation process. In the first step, all resulting stresses and strains for each finite shell element are computed by LAGRANGE and passed to the PSDA strength routine. Hereby, stress based failure initiation criteria

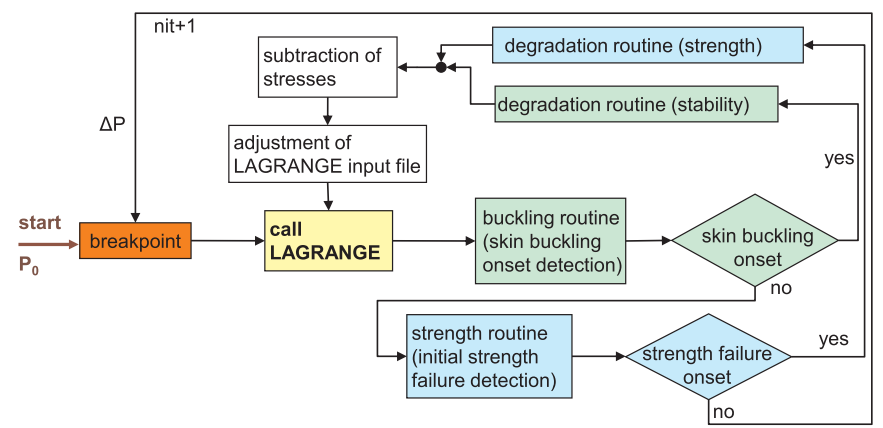

Fig. 6. PSDA process. 


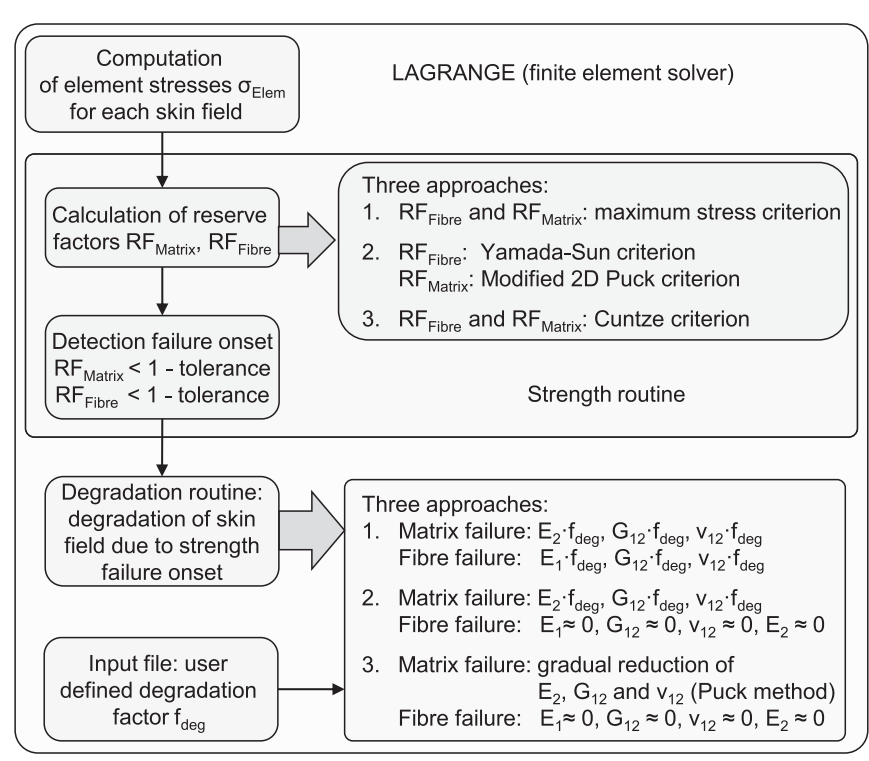

Fig. 7. Detection of strength failure and degradation process within the PSDA.

are used to calculate the reserve factors for fibre $\left(\mathrm{RF}_{\mathrm{Fibre}}\right)$ and matrix failure $\left(\mathrm{RF}_{\text {Matrix }}\right)$ onset of an unidirectional lamina. Delaminations are not considered. If the reserve factors are smaller than 1 minus a parameter "tolerance", which is defined within the input file, failure onset is detected. If the tolerance is greater than 0 , failure is detected at higher stresses.

Within the strength routine three stress based failure criteria are implemented. The first method computes matrix and fibre failure using the maximum stress criterion (see Nahas [31]). If the stress in fibre direction or matrix direction exceeds the corresponding allowable stress, the ply fails. Within the second method, the Yamada-Sun approach is used to calculate the reserve factor for fibre failure and the modified two dimensional Puck method computes the reserve factor for matrix cracking. The latter is a simplification of the two dimensional Puck theory, where only one failure mode is considered (see Calomfirescu et al. [14]). For the usage in the PSDA, additional parameters for the Puck criterion are taken from Puck et al. [37]. Within the third method, the two dimensional Cuntze criterion is used. The user has to choose which criterion is appropriate for the problem. To the authors knowledge a universally valid criterion does not exist (see Hinton et al. [23] and Soden et al. [42]).

If strength failure onset is detected in the second step, the strength degradation routine is performed where the ply properties can be reduced gradually or instantaneously. The first method implies instantaneous degradation. After the detection of fibre failure, the elastic modulus in fibre direction $E_{1}$, the in-plane shear modulus $G_{12}$ and the Poisson ratio $\nu_{12}$ are reduced instantaneously by a user defined factor $f_{\text {deg. }}$. When matrix failure occurs, the elastic modulus perpendicular to the fibre direction $E_{2}$, the shear modulus $G_{12}$ and the Poisson ratio $\nu_{12}$ are reduced by the factor $f_{\text {deg }}$. The elastic modulus in fibre direction $E_{1}$ remains constant. Both degradation factors are set within the input file. The second instantaneous degradation approach is similar to the first, but the respective ply fails completely, if fibre failure is determined. This means that $E_{1}, E_{2}, G_{12}$ and $\nu_{12}$ are set to nearly 0 . Within the third gradual degradation approach the Puck degradation method (see [35]) is used. The Puck method computes a degradation factor using parameters adjusted by test results (see [24]). It is used to reduce the ply properties gradually. For more information regarding the degradation approaches, please refer to [21].

If no further damage is detected within the strength or buckling routines after the recalculation of the structure, the number of steps is increased and the next load step is performed. The PSDA terminates, if one exit condition, which is checked at the "breakpoint" (see Fig. 6) is
Table 1

Input parameters.

\begin{tabular}{ll}
\hline Input parameter & Value \\
\hline Stacking sequence & {$[45-450900900-4545]$} \\
Ply thickness & $0.12 \mathrm{~mm}$ \\
Material parameter & $\mathrm{E}_{1}=157,000 \mathrm{~N} / \mathrm{mm}^{2} ; \mathrm{E}_{2}=$ \\
& $8500 \mathrm{~N} / \mathrm{mm}^{2} ;$ \\
& $\mathrm{G}_{12}=4200 \mathrm{~N} / \mathrm{mm}^{2} ; \nu_{12}=0.35$ \\
Dimension in x-direction & $1920 \mathrm{~mm}$ \\
Dimension in y-direction & $600 \mathrm{~mm}$ \\
User defined factor $\mathrm{f}_{\text {Post }}$ & $\mathrm{f}_{\text {Post }}=1.1$ \\
User defined factor $\mathrm{f}_{\text {fail }}$ calculating the & $\mathrm{f}_{\text {fail }}=1.6$ \\
$\quad$ critical load $\mathrm{P}_{\text {fail }}$ & \\
\hline
\end{tabular}

active. This includes the maximum number of iterations, and the fact that all skin fields have degraded due to skin buckling or if strength failure occurs.

\section{Application of the PSDA}

\subsection{Model}

In this section, an example with 16 skin fields illustrates the buckling analysis within the PSDA. The resulting load displacement curves and slopes are compared to the ones computed by the nonlinear ABAQUS analyses to verify the element stiffness reduction due to skin buckling. Since the prediction and degradation due to strength failure is verified within numerous state of the art publications. Therefore, the next section focuses on skin buckling onset.

All 16 skin fields, which are meshed with finite shell elements, are flat, non-stiffened and simply supported. The latter means that the displacements and their rotations are prevented at all edges of each skin field. The input data for all skin fields is summarised in Table 1. The material data, element dimensions, ply thickness and stacking sequences are commonly used in industrial applications. As already mentioned the factors $\mathrm{f}_{\text {Post }}$ and $\mathrm{f}_{\mathrm{Fail}}$ have to be chosen between 1.1 and 1.6, whereas $f_{\text {Fail }}$ has to be greater than $f_{\text {Post }}$. The factor $f_{\text {Fail }}$ has the value 1.6 to cover a large post buckling area. To investigate the influence of $\mathrm{f}_{\text {Post }}$ on the resulting load displacement curves, several PSDA and ABAQUS analyses were performed using 1 skin field. Regarding uniaxial compression $f_{\text {Post }}$ has only a small influence on the load displacement curve. This is different for shear loading. For $\mathrm{f}_{\text {Post }}>1.1$ the residual element stiffness of the degraded skin field is too small in the post buckling regime, which leads to a large deviation of the PSDA and ABAQUS slope. Therefore, the value for $\mathrm{f}_{\text {Post }}$ is 1.1.

The ABAQUS model, the PSDA model and the loading condition are illustrated in Fig. 8. As already mentioned, LAGRANGE within the PSDA calculates skin buckling onset using the Weaver and ESDU method. Both methods assume simply supported skin fields and each skin field is computed separately.

The skin fields within the ABAQUS model have to depict the same behaviour in order to compare both load displacement curves and slopes. Therefore, additional equations are needed in ABAQUS, which are explained in [21]. Those equations ensure that the lines parallel to the $\mathrm{x}$-axis deform in a linear pattern within the ABAQUS model similar to the skin fields in the PSDA, which are meshed with only one shell element. To ensure the same loading condition for the PSDA and the ABAQUS model, an additional ABAQUS model is generated with the
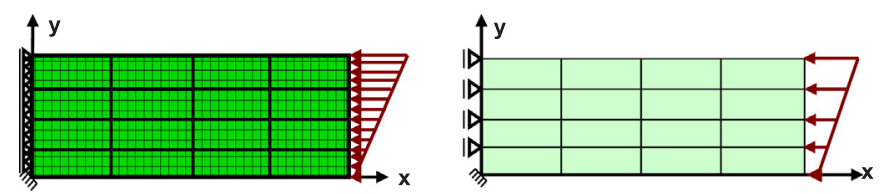

Fig. 8. 16 skin field example for PSDA (right) and ABAQUS (left) (see [21]). 

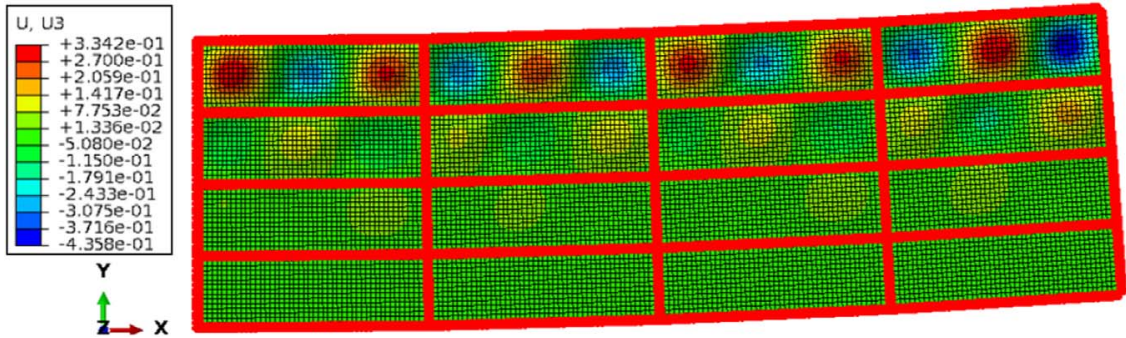

Fig. 9. Skin buckling onset of 16 skin field example (see [21]).

same number of finite shell elements as the coarse PSDA model. The resulting reaction forces from the coarse ABAQUS model are then applied to the PSDA model. This approach is possible, since the four node shell elements within ABAQUS and LAGRANGE use a linear displacement function.

\subsection{Results}

Skin buckling onset ensues in the upper four elements of the 16 skin elements (Fig. 9).

The resulting load displacement curves from the ABAQUS analysis and the PSDA are illustrated in Fig. 10. The purple curve represents the load displacement curve resulting from ABAQUS without buckling failure. This curve allows determining skin buckling onset with more ease. It is visible, that the PSDA detects skin buckling onset prior to ABAQUS. Regarding the stresses within the elements (see also Fig. 9) the values of the stresses within the ABAQUS analysis are almost the same at the point where the PSDA detects skin buckling onset. The reason for the deviation in loads and stresses is the conversion of the ABAQUS loads into the PSDA model nodal forces. To compute the nodal forces, the stresses within the ABAQUS model are computed at the integration points of the finite shell element and multiplied by the corresponding areas. Afterwards, the resulting forces are extrapolated to the corresponding nodes. This causes differences between the stress contour plot for the coarse PSDA (LAGRANGE) and ABAQUS model. After the critical load Pfail, which has the value $6200 \mathrm{~N}$, the slope resulting from the PSDA drops significantly, since the material properties of the shell elements, where final buckling failure is detected, are set to nearly zero.

To quantify the deviation between both analyses, the slopes are compared for three different displacements. The first point is located right after skin buckling onset at $0.46 \mathrm{~mm}$. Here both slopes differ by $15 \%$ due to the instantaneous stiffness reduction within the PSDA. Between skin buckling onset and complete failure of the first finite shell element $(0.5 \mathrm{~mm}$ and $0.53 \mathrm{~mm})$, the difference between both slopes has a value of about $11 \%$. Before the first element fails completely $(0.56 \mathrm{~mm})$, the difference between the PSDA and the ABAQUS slope is $7.8 \%$. Since the slope resulting from the PSDA simplifies the post buckling regime, the nonlinear ABAQUS analysis is more accurate than the PSDA. However, when using ABAQUS the computational costs would be too high within the preliminary design process, especially when optimising large aircraft structures. Not every physical detail is modelled here to ensure a fast optimisation with a large number of variations. Therefore, the PSDA should be used at the preliminary design stage. Despite the deviations, the behaviour in the post buckling regime is modelled with sufficient accuracy and the buckling load is underestimated compared to the ABAQUS analyses leading to a more conservative design. Regarding the 16 skin field example, the PSDA is 6 times faster than the nonlinear ABAQUS analysis. Whereas ABAQUS requires 100 iterations and a total time of $1449 \mathrm{~s}$, the PSDA computes the results within only $233 \mathrm{~s}$ using 140 load steps.

The overall computational costs do not include the preliminary nonlinear ABAQUS analysis, which are used to compute the degradation factor for each individual skin field and therefore, enhance the resulting slope of the PSDA. Without the ABAQUS analyses, the PSDA could not depict the post buckling regime. However, this nonlinear analysis has to be done only once for each individual skin field and the results can be used for each skin field with the same characteristics. In the present section all 16 skin fields had the same element properties. Also the same loading condition is applied. Therefore, the preliminary ABAQUS analysis is performed only once for the whole 16 skin field example.

\section{Conclusion}

This paper presented a progressive stiffness degradation analysis (PSDA), which enables the prediction of the structural behaviour after strength failure initiation and skin buckling onset using closed form

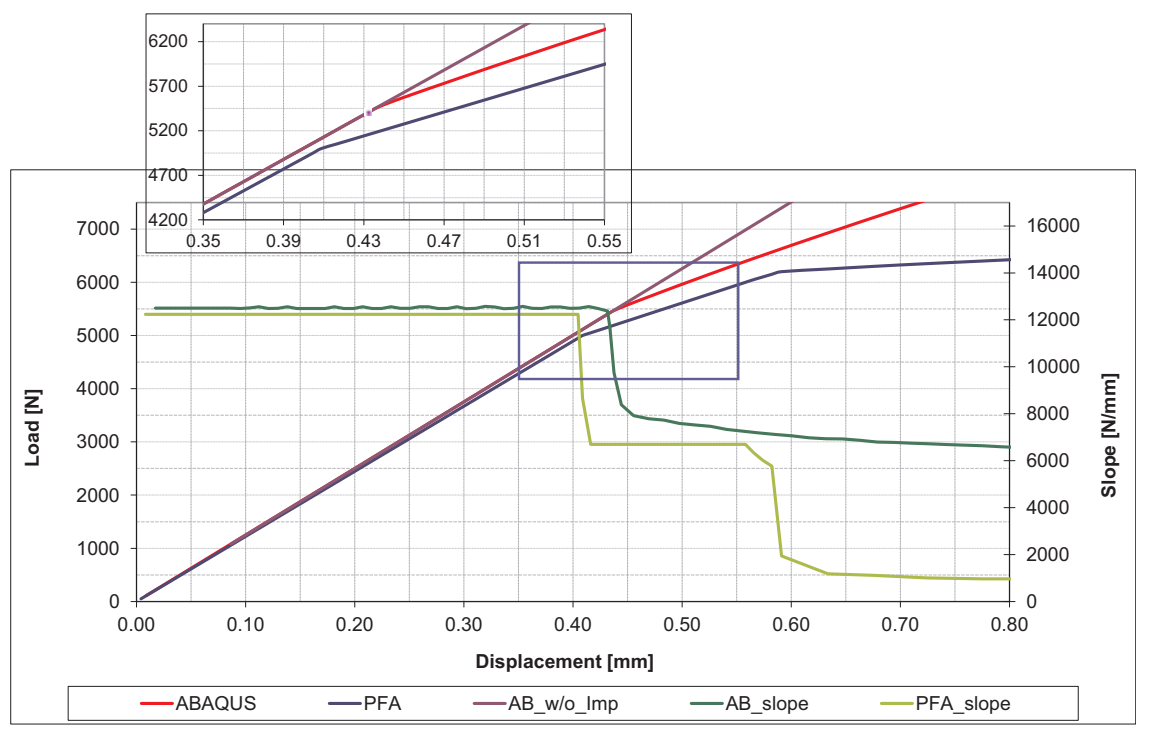

Fig. 10. Comparison of load displacement curves for 16 skin field example (see [21]). 
solutions. In the first part of this paper, several strength failure initiation criteria and degradation methods are reviewed. Also the prediction of buckling failure within composites is briefly discussed. The PSDA process and the integration of the buckling behaviour are described. Within the PSDA, the user can choose between different state of the art strength failure initiation and degradation methods. After the detection of skin buckling onset, the membrane stiffness matrix of the corresponding finite element is multiplied by a degradation factor, which is computed from several ABAQUS analyses. Hereby, only a part of the complete load displacement behaviour in the post buckling regime is considered, which introduces a simplified approach when considering the post buckling behaviour of composite structures. Therefore, for verification purposes, an example is computed using the PSDA and nonlinear ABAQUS analysis. It consists of 16 finite shell elements. The resulting load displacement curves and slopes from the PSDA are compared to the ones from the ABAQUS analysis. When comparing the PSDA with the ABAQUS analysis, deviations are visible in the resulting slopes when the PSDA detects complete failure within one finite shell element. Here the entries of the corresponding stiffness matrix are set to nearly 0 and the PSDA slope drops significantly. However, despite the deviations it can be said that the PSDA reproduces the nonlinear post buckling regime with sufficient accuracy. Additionally, the PSDA is about 6 times faster than the corresponding ABAQUS analysis.

Certain limitations arise due to the fact that the finite element solver within LAGRANGE is used to calculate the load where skin buckling onset is detected. For example curved shell elements are not supported and global buckling is neglected. Additionally, this paper only considers the analysis of membrane loading conditions, which excludes delaminations between the plies. Nevertheless, when using fast methods, especially when optimising large structures within the preliminary design process of aircrafts, it is not necessary to model all physical details.

\section{Acknowledgments}

The author would like to thank the colleagues from Airbus Defense \& Space for providing the software LAGRANGE. The author also would like to thank Prof. Dr.-Ing. M. Wiedemann and Prof. Dr.-Ing. habil. K. Rohwer for the many fruitful discussions.

\section{References}

[1] S. Adden, P. Horst, Stiffness degradation under fatigue in multiaxially loaded noncrimped-fabrics, Int. J. Fatigue 32 (1) (2010) 108-112.

[2] H. Altenbach, J. Altenbach, R. Rikards, Einführung in die Mechanik der Laminatund Sandwichtragwerke, Deutscher Verlag für Grundstoffindustrie, Stuttgart, 1996.

[3] K.N. Anyfantis, N.G. Tsouvalis, Post buckling progressive failure analysis of composite laminated stiffened panels, Appl. Compos. Mater. 19 (3-4) (2011) 219-236.

[4] S. Barakat, G.A. Abu-Farsakh, The use of an energy-based criterion to determine optimum configurations of fibrous composites, Compos. Sci. Technol. 59 (12) (1999) 1891-1899.

[5] L. Boni, Fantera, D. Lanciotti, A, post-buckling behavior of flat stiffened composite panels: experiments vs. analysis, Compos. Struct. 94 (12) (2012) 3421-3433.

[6] G.H. Bryan, On the stability of a plane plate under Thrusts in its own plane, with applications to the buckling of the sides of a ship, Proc. Lond. Math. Soc. 1-22 (1) (1980) 54-67.

[7] P. Buermann, R. Rolfes, J. Teßmer, M. Schagerl, A semi-analytical model for local post-buckling analysis of stringer- and frame-stiffened cylindrical panels, ThinWalled Struct. 44 (1) (2006) 102-114.

[8] D. Bushnell, Stress, stability and vibration of complex, branched shells of revolution, Comput. Struct. 4 (1974) 399-435.

[9] D. Bushnell, Bosor5 - Program for buckling of elastic-plastic complex shells of revolution including large deflections and creep, Comput. Struct. 6 (1975) 221-239.

[10] D. Bushnell, Panda - Interactive program for minimum weight design of stiffened cylindrical panels and shells, Comput. Struct. 16 (1-4) (1983) 167-185.

[11] D. Bushnell, W.D. Bushnell, Minimum-weight design of a stiffened panel via PANDA2 and evaluation of the optimized panel via STAGS, Comput. Struct. 50 (4) (1994) 569-602.

[12] R. Butler, F.W. Williams, Optimum design using VICONOPT, a buckling and strength constraint program for prismatic assemblies of anisotropic plates, Comput. Struct. 43 (4) (1991) 699-708.

[13] E. Byklum, E. Steen, J. Amdahl, A semi-analytical model for global buckling and post buckling analysis of stiffened panels, Thin-Walled Struct. 42 (5) (2004)
$701-717$

[14] M. Calomfirescu, F. Daoud, T. Pühlhofer, A new look into structural design philosophies for aerostructures with advanced optimisation methods and tools, in: IV European Conference on Computational Mechanics, France, 2010.

[15] J.N. Craddock, D.J. Champagne, A comparison of failure criteria for laminated composite, AIAA 85/0739, AIAA/ASME/ASCE/AHS, in: Proceedings of the 23rd Structures, Structural Dynamics and Materials Conference, 1982

[16] R.G. Cuntze, Efficient 3D and 2D failure conditions for UD laminae and their application within the verification of the laminate design, Compos. Sci. Technol. 66 (7-8) (2006) 1081-1096.

[17] F. Daoud, M. Calomfirescu, Optimisation of composite aircraft structures in consideration of post buckling behavior, Int. J. Struct. Stab. Dyn. 10 (905) (2010).

[18] Buckling of flat rectangular plates, ESDU 81047, Engineering Sciences Data Unit, 1994.

[19] F. Daghia, P. Ladevèze, A micro-meso computational strategy for the prediction of the damage and failure of laminates, Compos. Struct. 94 (12) (2012) 3644-3653.

[20] M. Fischer, D. Kennedy, C.A. Featherson, Multilevel Optimization of Aerospace and Lightweight Wings, 23rd International Congress of Aeronautical Sciences, Toronto, Canada, 2002.

[21] T. Führer, Composite structures' stiffness degradation due to strength and stability (Ph.D. Dissertation), DLR Bibliotheks- und Informationswesen, Köln, 2013.

[22] M.R. Garnich, V.M.K. Akula, Review of Degradation Models for Progressive Failure Analysis of Fibre Reinforced Polymer Composites, ASME, 2009.

[23] M.J. Hinton, A.S. Kaddour, P.D. Soden, A comparison of the predictive capabilities of current failure theories for composite laminates, judged against experimental evidence, Compos. Sci. Technol. 62 (12-13) (2002) 1725-1797.

[24] M. Knops, C. Bögle, Gradual failure in fibre/polymer laminates, Compos. Sci. Technol. 66 (5) (2006) 616-625.

[25] M. Kober, Ein Beitrag zur strukturmechanischen Optimierung realer Bauteile in metallischer und Faser-verbundbauweise unter Verwendung von wirkebenenbasierten Bruchkriterien, Mensch \& Buch, Januar, 2012.

[26] W.T. Koiter, On the stability of elastic equilibrium, (in Dutch), (Ph.D. Dissertation), Delft University of Technology, 1945, Englisch Translation by E. Riks: A Translation of the Stability of Elastic Equilibrium, Stanford University, 1970.

[27] N. Kroll, M. Abu-Zurayk, D. Dimitrov, T. Franz, T. Führer, T. Gerhold, S. Görtz, R. Heinrich, C. Ilic, J. Jepsen, J. Jägersküpper, M. Kruse, A. Krumbein, S. Langer, D. Liu, R. Liepelt, L. Reimer, M. Ritter, A. Schwöppe, J. Scherer, F. Spiering, R. Thormann, V. Togiti, D. Vollmer, J.-.H. Wendisch, DLR-Projekt Digital-X auf dem Weg zur virtuellen Flugzeugentwicklung und Flugerprobung auf Basis höherwertiger Verfahren, DLRK2014-340099, Deutscher Luft- und Raumfahrtkongress, 2014.

[28] R. Krueger, The Virtual Crack Closure Technique: History, Approach and Applications, NASA/CR-2002-211628, ICASE Report No. 2002-2010, 2002.

[29] P. Kuhn, J.P. Peterson, L.R. Levin, A summary of diagonal tension, part I - methods and analysis, Technical Note 2661, NACA, 1952.

[30] Quick LAGRANGE, Reference guide version 1.1.0, European Aeronautic defence and space company CASSIDIAN, Dep. COEAC5 - Des. Autom. Optim. (2011).

[31] M.N. Nahas, Survey of failure and post-failure theories of laminates fibre-reinforced composites, J. Compos. Technol. Res. 8 (4) (1986) 138-153.

[32] S. Oh, K. Kim, S. Kim, An efficient post buckling analysis technique for composite stiffened curved panels, Compos. Struct. 74 (3) (2006) 361-369.

[33] P. Pevzner, H. Abramovich, T. Weller, Calculation of the collapse load of an axially compressed laminated composite stringer-stiffened curved panel-An engineering approach, Compos. Struct. 83 (4) (2008) 341-353.

[34] E.A. Phillips, C.T. Herakovich, L.L. Graham, Damage development in composite with large stress gradients, Compos. Sci. Technol. 61 (15) (2001) 2169-2182.

[35] A. Puck, Festigkeitsanalyse von Faser-Matrix-Laminaten, Carl Hanser Verlag, München Wien, 1996.

[36] A. Puck, H. Schürmann, Failure analysis of FRP laminates by means of physically based phenomenological models, Compos. Sci. Technol. 58 (7) (1998) 1045-1067.

[37] (a) A. Puck, J. Kopp, M. Knops, Guidelines for the determination of the parameters in Puck's action plane strength criterion, Composites Science and Technology, 62 (3) (2002) 371-378;

(b) A. Puck, J. Kopp, M. Knops, Errata to 'Guidelines for the determination of the parameters in Puck's action plane strength criterion', Compos. Sci. Technol. 62 (9) (2002) 1275

[38] Y.S. Reddy, J.N. Reddy, Three-dimensional finite element progressive failure analysis of composite laminates under axial extension, J. Compos. Technol. Res. 15 (2) (1993) 73-87.

[39] Y.S.N. Reddy, C.M. Moorthy, J.N. Reddy, Non-Linear Progressive Failure Analysis of Laminated Composite Plates, Int. J. Non-Linear Mech. 30 (5) (1995) 629-649.

[40] K. Rohwer, Simulation of fibre composites: an assesment, in: M. Wiedemann, M. Sinapius (Eds.), Adaptive, Tolerant and Efficient Composite Structures, Springer Berlin, Heidelberg, 2012.

[41] G. Schuhmacher, M. Stettner, R. Zotemantel, O. O'Leary, M. Wagner, Optimisation assisted structural design of a new military transport aircraft, in: Proceedings of the 10th AIAA/ASSMO Multidisciplinary Analysis and Optimisation Conference, New York, 2004.

[42] P.D. Soden, A.S. Kaddour, M.J. Hinton, Recommendations for designers and researchers resulting from the world-wide failure exercise, Compos. Sci. Technol. 64 (3-4) (2004) 589-604.

[43] C.T. Sun, J. Tao, Prediction of failure envelopes and stress/strain behavior of composite laminates, Compos. Sci. Technol. 58 (7) (1998) 1125-1136.

[44] T. von Karman, H. Tsien, The buckling of thin cylindrical shells under axial compression, J. Aeronaut. Sci. 8 (8) (1941) 303-312.

[45] A. Watson, P.E. Fenner, C.A. Featherston, D. Kennedy, Postbuckled stability of 
panels with torsional buckling, in: Proceedings of the 50th AIAA/ASME/ASCE/ AHS/ASC Structures, Structural Dynamics \& Materials Conference, Palm Springs, USA, 2009.

[46] P. Weaver, An optimisation of long anisotropic flat plates subject to shear buckling loads, in: Proceedings of the 45th AIAA/ASME/ASCE/AHS/ASC Structures, Structural Dynamics \& Materials Conference, Palm Springs, USA, 2004.

[47] P. Weaver, Approximate analysis for buckling of compression loaded long rectangular plates with flexural/twist anisotropy, in: Proceedings of the Royal Society of London A: Mathematical, Physical and Engineering Sciences, Vol. 462, No. 2065, 2006, 59-73

[48] W. Wolfe, T. Butalia, A strain-energy based failure criterion for non-linear analysis of composite laminates subjected to biaxial loading, Compos. Sci. Technol. 58 (7) (1998) 1107-1124. 